\title{
Examples of nuclear systems
}

by

ED DUBINSKY* (Warszawa)

Abstract. The purpose of this paper is to present a detailed study of some concrete examples of nuclear systems, whose general theory has been presented by the author in previous papers. There are three classes of examples. First we consider nuclear systems generated either by a sequence of commuting normal operators or a sequence of permutations. Next, certain matrices which are zero everywhere except on the main diagonal and the diagonal directly above it are considered. Finally a very simple type of lower triancular matrix is discussed. In most cases it is shown that the resulting nuclear Fréchet space has a Schauder basis, but an example is constructed in which all of our methods fail to yield a basis.

The theory of nuclear systems (insofar as it has been developed) was presented in [1], [2]. This theory provides a method of constructing nuclear Fréchet spaces which in principle produces all such spaces whose topology is defined by norms and in practice permits the construction of examples not previously studied. Moreover, several criteria for the existence of Schauder bases have been established. It is the purpose of this paper to study in detail some of the examples of nuclear Fréchet spaces provided by nuclear systems, in most cases proving the existence of a Schauder basis.

We recall now the definitions and results which will be used. Proofs and further explanations are to be found in [1] and [2].

A nuclear system is a sequence $\left(A_{k}\right)$ of injective nuclear operators in $l_{2}$ with dense range. The associated space, written

$$
\hat{E}=\left\{\left(x_{k}\right): x_{k} \in l_{2}, x_{k}=A_{k}\left(x_{k+1}\right), k=1,2, \ldots\right\},
$$

is a subset of the countable product of copies of $l_{2}$ so it may be equipped with the subspace topology whence it becomes a nuclear Fréchet space

* Most of the research described in this paper was done during academic year 1969-70 while the author held a research associateship at McMaster University, Hamilton, Ontario. The opportunity was made possible, under extraordinary circumstances, through the efforts of B. Banaschewski and T. Husain. The author retains the deepest gratitude and highest respect for these colleagues, their department and McMaster University, whose actions in a crisis were exemplary. 
whose topology is defined by a sequence of norms, and all such spaces. are obtained in this way $([1]$, p. 373$)$. The projection operators, $P_{k}: \hat{\mathbb{E}} \rightarrow l_{2}$ $k=1,2, \ldots$ are defined by setting $P_{k}(x)=x_{k}$. Each $P_{l_{k}}$ is continuous, linear and has dense range $([1], p .376)$. We define $B_{0}$ to be the identity map on $l_{2}$ and, we set $B_{k}=A_{1} \ldots A_{k}, k_{k}=1,2, \ldots$

We recall that a Schauder basis in a topological vector space $Z$ is a sequence $\left(b_{n}\right)$ with the property that for each $x \in E$ there is a unique sequence $\left(\xi_{n}\right)$ of scalars such that $x=\lim _{n} \sum_{i=1}^{n} \xi_{i} b_{i}$. Wo shall call a sequence $\left(b_{n}\right)$ in a topological vector space total if the vector subspace it generates is dense.

For each positive integer $n$ we denote by $e^{n} \in l_{2}$ the sequence which is 1 at the $n$th term and 0 elsewhere and by $I_{n}$ the projection of an element of $l_{2}$ onto its $n$th coordinate. We denote by $p$ the subset of $l_{2}$ consisting of those sequences all but finitely many of whose terms are 0 .

A continuous linear map $D: l_{2} \rightarrow l_{2}$ is a diagonal map with diagonal element $\left(\lambda_{n}\right)$ if $D\left(e^{n}\right)=\lambda_{n} e^{n}, n=1,2, \ldots$ The identity map on $l_{2}$ will be denoted by $I$.

The following results from [1], [2] will be used quite often throughout the paper, so we quote them here for easy reference. Proposition $A$ is essentially proved in [1], p. 378 and appears in the following revised form as Theorem 3 in [2]. Propositions B, $\mathrm{C}$ are proved as Propositions 4,5 respectively in [2].

Proposition A. The associated space of a nuclear system $\left(A_{l_{k}}\right)$ has a Schauder basis if and only if there exist diagonal nuclear maps $D_{k}$ : $l_{2} \rightarrow l_{2}$, and continuous linear maps $f_{l k}: l_{2} \rightarrow l_{2}, k=1,2, \ldots$ such that

(i) $A_{k} f_{k+1}=f_{k} D_{l k}, k=1,2, \ldots$

(ii) $f_{1}$ maps $\bigcap_{k=1}^{\infty} D_{1} \ldots D_{k}\left(l_{2}\right)$ injectively onto $\bigcap_{k=1}^{\infty} A_{1} \ldots A_{k}\left(l_{2}\right)$.

Propositron B. If $\left(A_{k}\right)$ is a nuclear system, then $\hat{E}$ has a schauder basis if and only if there exists a linear injective map $s: \varphi \rightarrow \bigcap_{\gamma_{k}} B_{\psi_{k}}\left(l_{2}\right)$ with $B_{k}^{-1} S(\varphi)$ dense in $l_{2}$ for each $k \geqslant 0$ and such that for each $k \geqslant 0$ there exists $j \geqslant k$ such that

$$
\left.\sup _{p}|| B_{l_{c}^{-1}}^{-1} S I I_{p} S^{-1} B_{j}\right|_{B_{j}^{-1} S(p)} \|<\infty .
$$

In this case, if we consider $\hat{E}$ to be represented by $\bigcap_{k_{b}} B_{l_{c}}\left(l_{2}\right)$ (via $\left.P_{1}(\hat{E})\right)$, then the basis is the sequence $\left(S\left(e^{\nu}\right)\right)_{\nu}$.

Proposition C. Let $\left(A_{k}\right)$ be a nuclear system and $\left(b_{n}\right)$ a total, linearly independent sequence in $\hat{E}$. Then $\left(b_{n}\right)$ is a Schauder basis for $\hat{E}$ if and only if for each $k \geqslant 0$, there exists $j \geqslant k$ such that

$$
\sup _{p}\left\|\left.B_{k}^{-1} S \Pi_{p} S^{-1} B_{j}\right|_{B_{j}^{-1} S(\varphi)}\right\|<\infty
$$

where $S: \varphi \rightarrow l_{2}$ is defined by $S\left(e^{n}\right)=P_{1} b_{n}$.

In Section 1, we consider nuclear systems with the property that the eigenvectors of each $A_{k}$ can be easily computed and have a relatively transparent dependence on $k$. In Section 2, we consider nuclear systems generated by a single operator, $A$, that is, $A_{k}=A$ for all $k$, where $A$ is a matrix whose terms are 0 everywhere except the main diagonal and the diagonal just above it. We are able to give sufficient conditions for the existence of a basis and also construct an example in which two methods for obtaining a basis fail. This leads to an example of a Markuschevitch basis in a Fréchet nuclear space which is not a Schauder basis. In Section 3 we consider $A_{k}$ to be a matrix which is 0 except on the main diagonal and the first $N$ columns ( $N$ independent of $k$ ). Here $\hat{E}$ always has a basis.

1. Normal operators and permutations. In the next proposition we give a generalization of the result in [4]. The idea is that if the operators in a nuclear system all have the same set of eigenvectors, then this set can be used to construct a basis for the associated space.

Proposition 1. Let $\left(A_{k}\right)$ be a nuclear system in which each $A_{k}$ is normat and $A_{k} A_{k+1}=A_{k+1} A_{k}$ for all $k$. Then the associated space possesses a Schauder basis.

Proof. Let $\lambda_{1}$ be an eigenvector of $A_{1}$ with eigenspace $E_{1}$ which is finite dimensional since $A_{1}$ is compact. If $x \in E_{1}$ then $A_{1} A_{2}(x)=A_{2} A_{1}(x)$ $=\lambda_{1} A_{2}(x)$ so $A_{2}(x) \in E_{1}$. Thus $A_{2}\left(E_{1}\right) \subset E_{1}$ and $A_{2} \mid E_{1}$ is a normal operator on $E_{1}$ so we can choose a maximal eigenspace $E_{2} \subset E_{1}$ whose dimension is positive. Repeating the process indefinitely, we obtain a decreasing sequence $\left(E_{k}\right)$ of finite dimensional spaces with positive dimension and hence there exists $k_{0}$ such that $E_{k}=E_{k_{0}}$ for all $k \geqslant k_{0}$. It then follows that an orthonormal basis for $E_{k_{0}}$ is a non-empty set whose elements are eigenvectors for each $A_{k}, k=1,2, \ldots$

Repeating the process a number of times at most equal to the dimension of $E_{1}$, we obtain an orthonormal basis for $E_{1}$ whose elements are eigenvectors for each $A_{k}$. Again repeating for each eigenvalue of $A_{1}$, we obtain an orthonormal basis $\left(x_{n}\right)$ for $l_{2}$ such that each $x_{n}$ is an eigenvector of each $A_{k}$.

Finally, define $S: l_{2} \rightarrow l_{2}$ by $S\left(e^{n}\right)=x_{n}$ and apply Proposition B. Clearly $S(\varphi) \subset B_{k}\left(l_{2}\right)$ and $B_{k}^{-1} S(\varphi)$ is dense in $l_{2}$ for each $k$. Moreover 
we have $A_{k}=S D_{k} S^{*}$ where $D_{k}$ is a diagonal operator so $B_{k}=S D_{1} \ldots D_{k} S^{*}$ and so we have,

$$
\sup _{p}\left\|B_{k}^{-1} S \Pi_{p} S^{-1} B_{k}\right\|=\sup _{p}\left\|S\left(D_{1} \ldots D_{l_{k}}\right)^{-1} I_{p} D_{1} \ldots D_{k} S^{*}\right\| \leqslant\left\|S S^{*}\right\|=1
$$

so Proposition B applies to yield the desired result.

In view of Proposition 1 one might try to construct a nuclear Fréchet space without a basis by making the eigenvectors of the maps $A_{l}$ different for each $k$. This is perhaps also suggested by the proof of Proposition 2 of [2]. However this does not seem to work as is indicated by the next result in which we construct a basis in cases for which the eigenvectors are quite different.

Proposition 2. Let $\left(\sigma^{k}\right)$ be a sequence of permutations of the natural numbers and let $\left(\alpha^{k}\right)$ be a sequence of elements of $l_{1}$ each of which has no 0 terms. Define $A_{k}: l_{2} \rightarrow l_{2}$ by

$$
A_{k}\left(e^{n}\right)=\alpha_{n}^{k} e^{\sigma^{k}(n)}, \quad n, k=1,2, \ldots
$$

Then $\left(A_{k}\right)$ is a nuclear system whose associated space has a Schauder basis.

Proof. It is clear that $\left(A_{l_{t}}\right)$ is a nuclear system. To see that it has a basis, we apply Proposition A. Let $\tau^{k}=\sigma^{1} \ldots \sigma^{k}, \tau^{0}=$ identity and define diagonal operators $D_{k}: l_{2} \rightarrow l_{2}$ and continuous operators $f_{k}$ : $l_{2} \rightarrow l_{2}$ by

$$
D_{k}\left(e^{\tau^{k}(n)}\right)=\alpha_{n}^{k} e^{\tau^{k}(n)}, \quad f_{k}\left(e^{\tau^{k n-1}(n)}\right)=e^{n}, \quad k, n=1,2, \ldots
$$

Then we have

$$
\begin{aligned}
A_{k k} f_{k+1}\left(e^{\tau^{k}(n)}\right) & =A_{k}\left(e^{n}\right)=\alpha_{n}^{k} e^{\sigma^{k}(n)}=\alpha_{n n}^{k} f_{k}\left(e^{\tau^{k-1}\left(\sigma^{k}(n)\right)}\right) \\
& =f_{k}\left(\alpha_{n}^{k} e^{\tau^{k}(n)}\right)=f_{k} D_{k}\left(e^{\tau^{k}(n)}\right),
\end{aligned}
$$

so that $A_{k} f_{k+1}=f_{k} D_{k}, k=1,2, \ldots$

Finally; define $\beta^{k} \in l_{1}, l_{k}=1,2, \ldots$ by $\beta_{\tau^{k}(n)}^{k^{k}}=a_{n}^{k}$ so that $D_{k_{k}}\left(l_{2}\right)$ $=\beta^{k} l_{2}, \beta^{k}=\left(\beta_{n}^{k}\right)_{n}$.

Then we have,

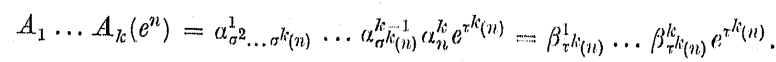

Hence, using the fact that $l_{2}$ is invariant under permutations,

$$
\begin{aligned}
A_{1} \ldots A_{k}\left(l_{2}\right) & =\left\{\sum_{n=1}^{\infty} \xi_{n} A_{1} \ldots A_{k}\left(e^{n}\right): \xi=\left(\xi_{n}\right) \in l_{2}\right\} \\
& =\left\{\sum_{n=1}^{\infty} \xi_{n} \beta_{\tau}^{1}{ }^{k}(n) \ldots \beta_{\tau}^{k} k_{(n)} e^{\tau^{k}(n)}: \xi \in l_{2}\right\} \\
& =\left\{\sum_{n=1}^{\infty} \xi_{n} \beta_{n}^{1} \ldots \beta_{n}^{k} e^{n}: \xi \epsilon l_{2}\right\}=\beta^{1} \ldots \beta^{k}\left(l_{2}\right)=D_{1} \ldots D_{k}\left(l_{2}\right) .
\end{aligned}
$$

Hence, $f_{1}\left(\bigcap_{k=1}^{\infty} D_{1} \ldots D_{k}\left(l_{2}\right)\right)=\bigcap_{k=1}^{\infty} D_{1} \ldots D_{k}\left(l_{2}\right)=\bigcap_{k=1}^{\infty} A_{1} \ldots A_{k}\left(l_{2}\right)$ so the conditions of Proposition A are satisfied and we may conclude that the associated space has a basis.

Remark 1. A slight improvement of Proposition 1 is possible. If we assume that each $A_{k}$ is similar to a diagonal matrix via the same similarity transformation; that is, there exists an isomorphism $S: l_{2} \rightarrow l_{2}$ such that each $S^{-1} A_{k} S$ is a diagonal matrix, then the last half of the proof of Proposition 1 will still work showing that $\hat{E}\left(A_{k}\right)$ was a basis.

2. $\mu-\nu$ matrices. Let $\mu, \nu$ be elements of $l_{1}$ with $0<\left|v_{n}\right| \leqslant\left|\mu_{n}\right|$ and define $A: l_{2} \rightarrow l_{2}$ by

$$
A\left(e^{\mathrm{I}}\right)=\mu_{1} e^{\mathrm{I}}, \quad A\left(e^{n}\right)=\mu_{n} e^{n}-v_{n-1} e^{n-1}, \quad n>1 .
$$

Then $A(x)=0$ if and only if

$$
x_{n+1}=\frac{\mu_{n}}{v_{n}} x_{n}=\ldots=\frac{\mu_{n} \cdots \mu_{1}}{v_{n} \ldots v_{1}} x_{1},
$$

so that $\left|x_{n}\right| \geqslant\left|x_{1}\right|$ for all $n$ so if $x \in l_{1}$, then $x=0$. Thus $A$ is injective. Moreover, it is obvious that $A(\varphi)=\varphi$ so $A$ has dense range. Thus $A$ generates a nuclear system. We now wish to study the existence of a basis with various additional restrictions on $\mu, v$.

Proposition 3. Let $\mu=v$ and suppose that for each $k \geqslant 0,\left(\left|\mu_{p}\right|^{2 k} p\right)_{p}$ is unbounded. Let $\left(b_{n}\right)$ be the sequence in $\hat{E}$ defined by taking $b_{n}=\left(b^{k, n}\right)_{k}$, where $b^{k, n}=A^{-k+1}\left(e^{n}\right), n, k=1,2, \ldots$ Then $\left(b_{n}\right)$ is a total, linearly independent sequence in $\hat{E}$ which is not a Schauder basis for $\hat{E}$.

Proof. Since $A(\varphi)=\varphi$ it follows that the given expression establishes $\left(b_{n}\right)$ as a sequence in $\hat{E}$. If

$$
\sum_{i=1}^{n} t_{i} b_{i}=0
$$

then it follows by taking $k=1$ and evaluating the sum of sequences at its first coordinate that

$$
\sum_{i=1}^{n} t_{i} e^{i}=0
$$

which implies that $t_{1}=\ldots t_{n}=0$. Hence $\left(b_{n}\right)$ is linearly independent. Moreover, for each $k$, the vector subspace generated by $\left(b^{k, n}\right)_{n n}$ is $A^{-k+1}(\varphi)=\varphi$ which is dense in $l_{2}$ so it follows that $\left(b_{n}\right)_{n}$ is total in $\hat{\mathbb{E}}$.

To show that $\left(b_{n}\right)$ is not a basis we apply Proposition O. Clearly we have $B_{k}=A^{k}, S$ is the identity and $B_{k}^{-1} S(\varphi)=\varphi$ so we must show that for some $k \leqslant j$ that by choosing a suitable index $p$ we can make $\left\|\left.A^{-k} \Pi_{p} A^{j}\right|_{\varphi}\right\|$ 
arbitrarily large. We take $k=1$ and we compute the matrix representation,

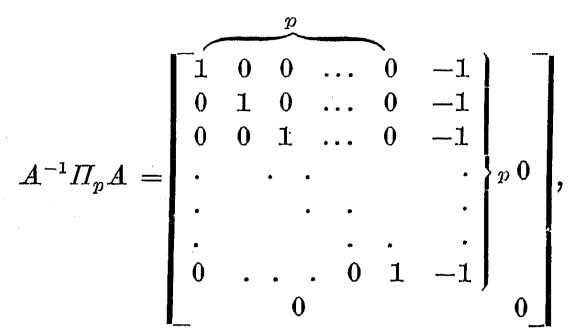

or, expressed in more compact form,

$$
A^{-1} \Pi_{p} A\left(e^{n}\right)=\left\{\begin{array}{cl}
e^{n}, & n \leqslant p \\
-\left(e^{1}+\ldots+e^{p}\right), & n=p+1 \\
0, & n>p+1
\end{array}\right.
$$

From this it follows that $\left\|\left.A^{-1} \Pi_{p} A\right|_{\varphi}\right\| \geqslant\left\|e^{1}+\ldots+e^{p}\right\|=\sqrt{p}$ so that the sequence is unbounded if we choose $j=1$.

Now choose any $j \geqslant 1$. We shall show that $\sup _{p}\left\|\left.A^{-1} I \Pi_{p} A^{j+1}\right|_{\varphi}\right\|=\infty$ which will complete the proof. Let the matrix of $A^{j}$ be $\left(a_{s t}^{j}\right)$, and take $p>j$. Then we claim

$$
a_{s, p+1}^{j}= \begin{cases}0 & \text { if } s \leqslant p-j \\ 0 & \text { if } s>p+1 \\ \mu_{p+1}^{j} & \text { if } s=p+1 .\end{cases}
$$

Indeed, if $j=1$ this is immediate from the definition. Suppose that it holds for some $j-1$. Then to compute the $(p+1)$-st column of $\left(a_{s t}^{j}\right)$ we multiply each row of $\left(a_{s t}^{1}\right)$ by the $(p+1)$-st column of $\left(a_{s t}^{j-1}\right)$. Thus we have,

$$
\begin{aligned}
a_{p+1, p+1}^{j} & =\sum_{n=1}^{\infty} a_{p+1, n}^{1} a_{n, p+1}^{j-1}=a_{p+1, p+1}^{1} a_{p+1, p+1}^{j-1}+a_{p+1, p+2}^{1} a_{p+-2, p+1}^{j-1} \\
& =\mu_{p+1} \mu_{p+1}^{i-1}-\mu_{p+1} \cdot 0=\mu_{p+1}^{j} .
\end{aligned}
$$

If $s>p+1$,

$$
a_{s, p+1}^{j}=\sum_{n=1}^{\infty} a_{s, n}^{1} a_{n, p+1}^{j-1}=\sum_{n=s}^{s+1} a_{s, n}^{1} a_{n, p+1}^{j-1}=0
$$

and if $s \leqslant p-j$,

$$
a_{s, p+1}^{j}=\sum_{n=1}^{\infty} a_{s, n}^{1} a_{n, p+1}^{j-1}=\mu_{s}^{1} a_{s, p+1}^{j-1}-\mu_{s}^{1} a_{s+1, p+1}^{j-1}
$$

and $s \leqslant p-j$ implies that $s \leqslant p-j+1$ and $s+1 \leqslant p-j+1$ so by the induction hypothesis, both terms vanish and $a_{s, p+1}^{j}=0$. Thus the claim is proved.

From this it follows that for some scalars $c_{p-j+1}, \ldots, c_{p}$ we have

so that

$$
A^{j}\left(e^{p+1}\right)=\sum_{s=p-j+1}^{p} c_{s} e^{s}+\mu_{p+1}^{j} e^{p+1}
$$

$$
\begin{aligned}
A^{-1} \Pi_{p} A^{j+1}\left(e^{p+1}\right) & =\sum_{s=p-j+1}^{p} c_{s} e^{s}-\mu_{p+1}^{j}\left(\sum_{s=1}^{p} e^{s}\right) \\
& =-\sum_{s=1}^{p-j} \mu_{p+1}^{j} e^{s}+\sum_{s=p-j+1}^{p}\left(c_{s}-\mu_{p+1}^{j}\right) e^{s}
\end{aligned}
$$

Therefore,

$$
\begin{aligned}
\left\|\left.A^{-1} \Pi_{p} A^{j+1}\right|_{\varphi}\right\| & \geqslant\left[\sum_{j=1}^{p-j}\left(\mu_{p+1}^{j}\right)^{2}+\sum_{s=p-j+1}^{p}\left(c_{s}-\mu_{p+1}^{j}\right)^{2}\right]^{\frac{1}{2}} \geqslant\left|\mu_{p+1}^{j}\right| \sqrt{p-j} \\
& =\left|\mu_{p+1}\right|^{j} \sqrt{p+1} \frac{\sqrt{p-j}}{\sqrt{p+1}} .
\end{aligned}
$$

By our hypotheses, this last term is unbounded in $p$ so we are finished.

Remark 1 . The hypothesis of Proposition 3 is easily obtained, for instance if we choose $\mu \in l_{1}$ such that

$$
\mu_{2^{2}}=\frac{1}{2^{n}}, \quad n=1,2, \ldots
$$

Then if we take $p=2^{2^{n}}$, we obtain

$$
\left|\mu_{p}\right|^{2 j} p=\frac{2^{2^{n}}}{2^{2 n j}} \rightarrow \infty \quad \text { as } n \rightarrow \infty .
$$

Remark 2. In a sense one may consider that Proposition 3 shows that the most naive approach to constructing a basis in a nuclear Fréchet space cannot in general succeed. Indeed we may think of $\hat{E}$ as a dense subspace of $l_{2}$ with a sequence of (Hilbertable) norms on it, the first being the original $l_{2}$ norm. Then we have taken a complete orthonormal sequence (with respect to the first norm) and shown that it was total and linearly independent in $\hat{E}$. Such a procedure is exactly what worked in the cases treated in Section 1 , but it fails here.

Actually, we can show more. Recall that a Markuschevich basis in a linear topological space $E$ is a sequence $\left(b_{n}, f_{n}\right)$, where $\left(b_{n}\right)$ is total in $E,\left(f_{n}\right)$ is total in $E^{\prime}\left[\mathfrak{T}_{s}(E)\right]$ and $f_{n}\left(b_{m}\right)=\delta_{n m}$. In [3] it is shown that if $E$ and its strong dual are separable then a Markuschevich basis always exists. A Markuschevich basis is not necessarily a Schauder basis. For an example in the case of Banach spaces, see [5]. We know of no previous example for Fréchet nuclear spaces, so we show that $\mu-v$ matrices provide such examples. 
Proposirron 4. With the hypotheses of Proposition 3 there is a sequence $\left(f_{n}\right)$ in $\hat{E}^{\prime}$ such that $\left(b_{n}, f_{n}\right)$ is a Markuschevich basis but not a Schauder basis.

Proof. For each $n=1,2, \ldots$ let $f_{n}=\Pi_{n} P_{1}$. Since $\Pi_{n}$ and $P_{1}$ are continuous it follows that $f_{n} \in \hat{E^{\prime}}$. Also,

$$
f_{n}\left(b_{m}\right)=\Pi_{n} P_{1}\left(b_{m}\right)=\Pi_{n}\left(b^{1, m}\right)=\Pi_{n}\left(e^{n}\right)=\delta_{n m} .
$$

Finally, if $x \in \hat{E}$ and $f_{n}(x)=0$ for all $n$, then $P_{1} x \in l_{2}$ so we can write

$$
P_{1} x=\sum_{m=1}^{\infty} \xi_{m} e^{m}, \quad \xi \in l_{2} \text { and convergence in } l_{2} \text {. }
$$

Hence, for each $n$,

$$
0=f_{n}(x)=\Pi_{n} P_{1}(x)=\sum_{m=1}^{\infty} \xi_{m} g_{n}\left(e^{m}\right)=\xi_{n}
$$

so it follows that $P_{1}(x)=0$ and since $P_{1}$ is injective, $x=0$. Thus $\left(f_{n}\right)$ is total in $E^{\prime}\left[\mathfrak{I}_{s}(E)\right]$ and we are finished.

We can try to find a basis for $\hat{E}$ using other methods. Let us assume for the rest of this section that $\mu_{n} \neq \mu_{m}$ for $n \neq m$. Then it is possible to (formally) diagonalize $A$. Let $D$ be the diagonal matrix with diagonal elements $\left(\mu_{n}\right)$ and let $U$ be the following matrix:

$$
\left[\begin{array}{ccccc}
1 \frac{\nu_{1}}{\mu_{1}-\mu_{2}} & \frac{\nu_{1} \nu_{2}}{\left(\mu_{1}-\mu_{3}\right)\left(\mu_{2}-\mu_{3}\right)} & \frac{\nu_{1} \nu_{2} \nu_{3}}{\left(\mu_{1}-\mu_{4}\right)\left(\mu_{2}-\mu_{4}\right)\left(\mu_{3}-\mu_{4}\right)} & \cdots \\
0 & 1 & \frac{\nu_{2}}{\mu_{2}-\mu_{3}} & \frac{\nu_{2} \nu_{3}}{\left(\mu_{2}-\mu_{4}\right)\left(\mu_{3}-\mu_{4}\right)} & \cdots \\
0 & 0 & 1 & \frac{\nu_{3}}{\mu_{3}-\mu_{4}} & \cdots \\
0 & 0 & 0 & 1 & \cdots \\
. & . & . & . & \cdots
\end{array}\right]
$$

One can easily verify by direct computation that $A U=U D$. Moreover, $U$ has a (formal) inverse, $U^{-1}$ given by the following matrix:

$\left[\begin{array}{cc}1 & \frac{v_{1}}{\mu_{2}-\mu_{1}} \\ 0 & 1 \\ 0 & 0 \\ 0 & 0 \\ 0 & \cdot\end{array}\right.$

and hence we can write $A=U D U^{-1}$. Indeed all of the above statements are rigorously true if we only apply the operators to $\varphi$. It would be quite easy (Remark 1 ) to conclude that $\hat{E}$ has a basis if we knew that $U$ was an isomorphism on $l_{2}$. Unfortunately, as we shall see below (Remark 3) this is not necessarily the case. However we can give a simple condition under which $U$ is an isomorphism. More detailed computations could lead to sharper results than the following.

Propositron 5. Let $\mu, v$ be such that $\mu_{n} \neq \mu_{m}$ for $n \neq m$ and

$$
r=\sup _{i<n}\left|\frac{v_{i}}{\mu_{i}-\mu_{n}}\right|<\frac{1}{2}
$$

Then $\hat{E}$ has a basis which is obtained by applying $P_{1}^{-1}$ to each of the columns of $U$ (as an element of $l_{2}$ ).

Proof. In view of Remark 1, we need only show that $U$ is an isomorphism. The explicit description of the basis follows from the application of Proposition B.

To show that $U$ is an isomorphism, let $S: l_{2} \rightarrow l_{2}$ be the operator defined by $S\left(e^{n}\right)=e^{n+1}$ and let $E_{n}: l_{2} \rightarrow l_{2}$ be the diagonal operator whose diagonal is given by the sequence

$$
\left(\frac{v_{m} \cdot v_{m+1} \ldots v_{m+n-1}}{\left(\mu_{m}-\mu_{m+n}\right) \ldots\left(\mu_{m+n-1}-\mu_{m+n}\right)}\right)_{m=1}^{\infty}
$$

Then clearly we have $U=I+\sum_{n=1}^{\infty} S^{n} E_{n}=I+U_{0}$. It suffices to show that $\left\|U_{0}\right\|<1$. But $\|S\|=1$ and $\left\|E_{n}\right\|$ is the maximum of the moduli in the given sequence, that is,

$$
\left\|E_{n}\right\|=\sup _{m}\left|\frac{v_{m} \ldots v_{m+n-1}}{\left(\mu_{m}-\mu_{m+n}\right) \ldots\left(\mu_{m+n-1}-\mu_{m+n}\right)}\right|=\sup _{m} \prod_{i=m}^{m+n-1}\left|\frac{v_{i}}{\mu_{i}-\mu_{m+n}}\right| \leqslant r^{n} \text {. }
$$

Hence we have,

$$
\left\|U_{0}\right\| \leqslant \sum_{n=1}^{\infty}\left\|S^{n} E_{n}\right\| \leqslant \sum_{n=1}^{\infty}\left\|E_{n}\right\| \leqslant \sum_{n=1}^{\infty} r^{n}<\sum_{n=1}^{\infty} \frac{1}{2^{n}}=1 .
$$

Remark 3 . There are some simple cases in which the hypotheses of Proposition 5 are satisfied:

$$
\begin{aligned}
& \text { (i) } \mu_{n}=\frac{1}{n^{2}}, \quad \sup _{i} \frac{i^{2}(i+1)^{2}}{2 i+1}\left|v_{i}\right|<\frac{1}{2}, \\
& \text { (ii) } \mu_{n}=\frac{1}{2^{n}}, \quad \sup _{i} 2^{i}\left|v_{i}\right|<\frac{1}{4} .
\end{aligned}
$$

Returning now to the situation in Proposition 3, we may well ask if the 
method of Proposition 5 will always give a basis. The answer is no, and this will give us an example in which $U$ is not an isomorphism.

Proposition 6. Assume that $\mu=v, 0<\mu_{n} \neq \mu_{m}$ for $n \neq m$ and for each integer $j \geqslant 0$, the sequence

$$
\left(\frac{\mu_{p} \mu_{p+1}^{j}}{\mu_{p}-\mu_{p+1}}\right)_{p=1}^{\infty}
$$

is unbounded. Then the sequence $\left(b_{n}\right)$ given by $b_{n}=\mathcal{P}_{1}^{-1}\left(b^{1, n}\right)$, where $b^{1, n}$ is the sequence in the $n$-th column of $U$, is a total, linearly independent sequence in $\hat{E}$ which is not a Schauder basis.

Proof. Clearly $U(\varphi)=\varphi$ and $U$ is invective on $\varphi$. Hence $U\left(e^{n}\right) \in U(\varphi)$ $=U D^{k} U^{-1}(\varphi)=A^{k}(\varphi) \subset A^{k}\left(l_{2}\right)$ so $b^{1, n}=U\left(e^{n}\right) \in \bigcap_{k} A^{k}\left(l_{2}\right)$ and $b_{n} \in \hat{E}$. If $\sum_{i=1}^{n} t_{i} b_{i}=0$ then $\sum_{i=1}^{n} t_{i} U e^{i}=\sum_{i=1}^{n} t_{i} b^{1, i}=0$ and since $U$ is injective on $\varphi$, $\sum_{i=1}^{n} t_{i} e^{i}=0$ so $t_{1}=\ldots=t_{n}=0$ and $\left(b_{n}\right)$ is linearly independent. Finally, for each $k$, the subspace generated by $\left(b^{k, n}\right)_{n}$ is $A^{-k+1} U(\varphi)=\varphi$ so $\left(b_{n}\right)$ is total in $\hat{E}$.

Thus we may finish the proof by applying Proposition $\mathbf{C}$ to show that $\left(b_{n}\right)$ is not a Schauder basis. We shall show that the norm criteria is not satisfied for $k=1$. Let $j \geqslant 0$ and consider (all maps restricted to $\left.A^{-(j+1)} U(\varphi)=\varphi\right)$,

$$
A^{-1} U \Pi_{p} U^{-1} A^{j+1}=U D^{-1} \Pi_{p} D U^{-1} A^{i}=U \Pi_{p} U^{-1} A^{j} .
$$

Let $U \Pi_{p} U^{-1}=\left(\mu_{m n}\right)$ and we compute $\mu_{p, n}$ for $n=1,2, \ldots, p+1$. Now in $\Pi_{p} U^{-1}$, the first $p$ columns are the same as in $U^{-1}$ and the $(p+1)$ st column is the same as in $U^{-1}$ except the $(p+1)$ st row which is 0 instead of 1. Taking the inner product of each of these columns with the $p$ th row of $U$, we obtain,

$$
\mu_{p, n}=\left\{\begin{array}{cl}
0 & n<p \\
1 & n=p \\
-\frac{\mu_{p}}{\mu_{p}-\mu_{p+1}} & n=p+1 .
\end{array}\right.
$$

We have already partially computed the $(p+1)$ st column of $A^{j}=\left(a_{m n}^{j}\right)$ in the proof of Proposition 3 , but we also need to evaluate $a_{2,2 p+1}^{j}$. We claim

$$
a_{p, p+1}^{j}=-\mu_{p} \sum_{n=0}^{j-1} \mu_{p}^{n} \mu_{p+1}^{j-n-1}, \quad j \geqslant 1
$$

and $a_{p, p+1}^{0}=0$. This is clear for $j=1$ so we suppose it holds for $j$. Then using the information in the proof of Proposition 3, we obtain

$$
\begin{aligned}
a_{p, p+1}^{j+1} & =\sum_{i=1}^{\infty} a_{p i} a_{i, p+1}^{j}=\mu_{p} a_{p, p+1}^{j}-\mu_{p} \mu_{p+1}^{j} \\
& =-\mu_{p}\left(\mu_{p} \sum_{n=0}^{j-1} \mu_{p}^{n} \mu_{p+1}^{j-n-1}+\mu_{p+1}^{j}\right)=-\mu_{p} \sum_{n=0}^{j} \mu_{p}^{n} \mu_{p+1}^{j-n-1} .
\end{aligned}
$$

Hence we have,

$$
a_{m, p+1}^{j}=\left\{\begin{array}{cl}
-\mu_{p} \sum_{n=0}^{j-1} \mu_{p}^{n} \mu_{p+1}^{j-n-1}, & m=p, j \geqslant 1, \\
0, & m=p, j=0, \\
\mu_{p+1}^{j}, & m=p+1, j \geqslant 0, \\
0, & m>p+1, j \geqslant 0 .
\end{array}\right.
$$

Therefore we conclude that the element in the $p$ th row and $(p+1)$ st column of $A^{-1} U \Pi_{p} U^{-1} A^{j+1}$ is given by

$$
\begin{gathered}
-\mu_{p} \sum_{n=0}^{j-1} \mu_{p}^{n} \mu_{p+1}^{j-n-1}-\frac{\mu_{p} \mu_{p+1}^{j}}{\mu_{p}-\mu_{p+1}}, \quad j \geqslant 1, \\
-\frac{\mu_{p} \mu_{p+1}^{j}}{\mu_{p}-\mu_{p+1}}, \quad j=0 .
\end{gathered}
$$

Hence it follows that

$$
\left\|A^{-1} U \Pi_{p} U^{-1} A^{j+1}\right\| \geqslant\left\|A^{-1} U \Pi_{p} U^{-1} A^{j+1}\left(e^{p+1}\right)\right\| \geqslant \frac{\mu_{p} \mu_{p+1}^{j}}{\mu_{p}-\mu_{p+1}},
$$

and by hypothesis, this last sequence is unbounded with respect to $p$ for each $j \geqslant 0$ so the result follows from Proposition C.

Remark 4. It is important to note that there exist $\mu-\nu$ matrices which satisfy the hypotheses of both Proposition 3 and 6. Indeed, if we take $\mu$ such that

$$
\mu_{n}= \begin{cases}\frac{1}{2^{m}}, & n=2^{2^{m}} \\ \frac{1}{2^{m}}+\frac{1}{2^{m^{2}}}, & n=2^{2^{m}}-1,\end{cases}
$$

then (see Remark 1), the conditions of Proposition 3 are satisfied. Moreover, if we take any $j \geqslant 0$ and $p=2^{2^{n}}-1$, then

$$
\frac{\mu_{p} \mu_{p+1}^{j}}{\mu_{p}-\mu_{p+1}}=\frac{\left(\frac{1}{2^{m}}+\frac{1}{2^{m^{2}}}\right) \frac{1}{2^{j m}}}{\frac{1}{2^{m^{2}}}}=2^{m^{2}-m(j+1)}+\frac{1}{2^{j m}}
$$


which is clearly unbounded so Proposition 6 is satisfied. Thus all of the methods that we know of fail to produce a basis in this case and we are led to the following

CONJECTURE. If $A$ is a $\mu-\lambda$ matrix with $\mu=\lambda, 0<\mu_{n} \neq \mu_{m}$ for all $\dot{n} \neq m$ and such that $\mu$ satisfies (1), then $A$ generates a nuclear system whose associated space is a nuclear Irrechet space which does not have a Schauder basis.

3. Lower triangular matrices. In this section we consider a very simple case of lower triangular matrices which give nuclear systems and construct a basis by direct computation.

Let $N$ be a fixed positive integer and for each $k=1,2, \ldots$ let $a^{1, k}, \ldots, a^{N k}$ be elements of $l_{2}$ such that $a_{j}^{i / c}=0$ for $j \leqslant i$ and let $\mu^{k} \in l_{1}, \mu_{j}^{k} \neq 0$ for all $j, k$. Then we can define $A_{l_{k}}: l_{2} \rightarrow l_{2}$ by

$$
A_{k} x=\sum_{i=1}^{N} x_{i} a^{i, k}+\sum_{j=1}^{\infty} x_{j} \mu_{j}^{k} e^{j} .
$$

As a matrix we can describe $A_{l}$ by noting that $A_{k}$ is a diagonal matrix except for its $i$ th column $(1 \leqslant i \leqslant N)$ which is the sequence $\mu_{i}^{i c} e^{i}+a^{i k}$.

Proposition 7. $\left(A_{k}\right)$ is a nuclear system. For each $n$, the map $f_{n}=I I_{n} P_{1}$ is in the dual of $\hat{E}$.

Proof. Each $A_{k}$ is the sum of a nuclear diagonal map and a map with finite dimensional range so it is nuclear. If $A_{*}^{*}$ is the adjoint of $A_{k}$, then we can see by inspection that $A_{k}^{*}(\varphi)=\varphi$ so that $A_{k}^{*}$ has dense range so $A_{k}$ is injective. Next, it is clear that $e^{i}=\frac{1}{\mu_{i}^{k_{i}}} A_{k}\left(e^{i}\right)$ for $i>N$. For $1 \leqslant n \leqslant N$, let $\varepsilon>0$ and define $x=\left(x_{i}\right) \in l_{2}$ by

$$
x_{i}=\left\{\begin{array}{cl}
0, & i<n, \\
\frac{1}{\mu_{n}^{k}}, & i=n, \\
-\frac{1}{\mu_{i}^{k}}\left(a_{i}^{1, k} x_{1}+\ldots+a_{i}^{i-1, k c} x_{i-1}\right), & n<i \leqslant N, \\
-\frac{1}{\mu_{i}^{k}}\left(a_{i}^{1, k} x_{1}+\ldots+a_{i}^{N} x_{N}\right), & N<i \leqslant M, \\
0, & i>M,
\end{array}\right.
$$

where $M \geqslant N$ is an integer chosen such that

$$
\sum_{j=n}^{N}\left(\sum_{i=M+1}^{\infty}\left|a_{i}^{j, k} x_{j}\right|^{2}\right)^{\frac{1}{2}} \leqslant \varepsilon
$$

Then

$$
\left\|A_{k}(x)-e^{n}\right\|=\left\|\sum_{i=M+1}^{\infty}\left(a_{i}^{1, k} x_{1}+\ldots+a_{i}^{N, k} x_{N}\right) e^{i}\right\| \leqslant \varepsilon,
$$

so $A_{k}$ has dense range. Thus $\left(A_{k}\right)$ is a nuclear system.

The second statement is obvious since $\Pi_{n}, P_{1}$ are continuous.

Proposition 8. The associated space of $\left(A_{k}\right)$ has a Schauder basis.

Proof. For $i>N$ it follows that $e^{i} \in \bigcap_{k} A_{1} \ldots A_{k}\left(l_{2}\right)$ so we can define $b_{i}=P_{1}^{-1}\left(e^{i}\right) \epsilon \hat{E}$. Now $P_{1}(\hat{E})$ is a dense subspace of $l_{2}$ so if we define $\Pi: l_{2} \rightarrow R^{N}$ by $\Pi(x)=\left(x_{1}, \ldots, x_{N}\right)$ then $\Pi P_{1}(\hat{H})=R^{N}$. Hence there exists $b_{1}, \ldots, b_{N}$ in $\hat{E}$ such that $\Pi P_{1}\left(b_{i}\right)=e^{i} \in R^{N}, i=1, \ldots, N$.

We claim that $\left(b_{i}\right)$ is a Schauder basis for $\hat{E}$. First suppose that $x \in \hat{E}$ and $x=\sum \xi_{i} t_{i}$. Then by Proposition 7, for each $n, f_{n}(x)=\sum \xi_{i} f_{n}\left(b_{i}\right)$. Thus we obtain,

$$
\xi_{n}= \begin{cases}f_{n}(x) & n \leqslant N, \\ f_{n}(x)-\sum_{i=1}^{N} f_{i}(x) f_{n}\left(b_{i}\right) & n>N .\end{cases}
$$

This shows that the representation is unique and we need only show that for $x \in \hat{E}$, the series $\sum \xi_{i} b_{i}$ converges to $x$, where $\left(\xi_{i}\right)$ is given by the above relations.

We consider for $n=1,2, \ldots$

$$
\begin{aligned}
\Pi_{n} P_{1}\left(x-\sum_{i=1}^{N} \xi_{i} b_{i}\right) & =f_{n}(x)-\sum_{i=1}^{N} \xi_{i} f_{n}\left(b_{i}\right)=f_{n}(x)-\sum_{i=1}^{N} f_{i}(x) f_{n}\left(b_{i}\right) \\
& = \begin{cases}0, & n \leqslant N \\
\xi_{n}, & n>N\end{cases}
\end{aligned}
$$

and since $P_{1}\left(x-\sum_{i=1}^{N} \xi_{i} b_{i}\right) \in l_{2}$ we have $\xi \in l_{2}$ and

$$
P_{1}\left(x-\sum_{i=1}^{N} \xi_{i} b_{i}\right)=\sum_{n=N+1}^{\infty} \xi_{n} e^{n}
$$

Hence for $M \geqslant N$,

$$
\begin{aligned}
P_{1}\left(x-\sum_{i=1}^{M} \xi_{i} b_{i}\right) & =P_{1}\left(x-\sum_{i=1}^{N} \xi_{i} b_{i}\right)-\sum_{i=N+1}^{M} \xi_{i} P_{1}\left(b_{i}\right) \\
& =\sum_{n=N+1}^{\infty} \xi_{n} e^{n}-\sum_{i=N+1}^{M} \xi_{i} e^{i}=\sum_{n=M+1}^{\infty} \xi_{n} e^{n} .
\end{aligned}
$$


Now let $v_{n}^{k}=\mu_{n}^{1} \ldots \mu_{n}^{k}, \nu_{n}^{0}=1, n, k=1,2, \ldots$ Then for any $k \geqslant 1$, we have $\zeta^{k}=\left(\zeta_{n}^{k}\right)_{n} \in l_{2}$ with

so

$$
P_{k+1}\left(x-\sum_{i=1}^{M} \xi_{i} b_{i}\right)=\sum_{n=1}^{\infty} \zeta_{n}^{k} e^{n}
$$

$$
P_{1}\left(x-\sum_{i=1}^{M} \xi_{i} b_{i}\right)=A_{1} \ldots A_{k} P_{k+1}\left(x-\sum_{i=1}^{M} \xi_{i} b_{i}\right)=\sum_{n=1}^{\infty} v_{n}^{k} \xi_{n}^{k} e^{n} .
$$

Equating coefficients we conclude that

$$
\zeta_{n}^{k}=0 \text { for } n \leqslant M \quad \text { and } \quad \zeta_{n}^{k}=\frac{\xi_{n}}{\nu_{n}^{k}} \text { for } n>M .
$$

In particular, applying this for $M=N$ we conclude that

And for arbitrary $M \geqslant N$,

$$
\left(\frac{\xi_{n}}{\nu_{n}^{k}}\right)_{n=1}^{\infty} \epsilon l_{2} \text {. }
$$

$$
\left\|P_{k}\left(x-\sum_{i=1}^{M} \xi_{i} b_{i}\right)\right\|=\left\|\sum_{n=M+1}^{\infty} \frac{\xi_{n}}{v_{n}^{k}} e^{n}\right\|=\left(\sum_{n=M+1}^{\infty}\left|\frac{\xi_{n}}{v_{n}^{k}}\right|^{2}\right)^{\frac{1}{2}} .
$$

The last term goes to 0 as $M$ goes to $\infty$ and this implies

$$
\lim _{M \rightarrow \infty}\left\|P_{k}\left(x-\sum_{i=1}^{M} \xi_{i} b_{i}\right)\right\|=0 \quad \text { so that } \quad x=\sum_{i=1}^{\infty} \xi_{i} b_{i} .
$$

Remark 5. The case described above is a very primitive example. To go further, it would be very interesting to see what happens if $N$ varies with respect to $k$ and moreover if this approach could be used to approximate an arbitrary lower triangular matrix. Finally one could investigate the connection between upper and lower triangular matrices.

\section{References}

[1] Ed Dubinsky, Equivalent nuclear systems, Stud. Math., 38 (1970), pp. 373-379.

[2] - A new definition of nuolear systems with applisations to bases in muelear spaces Studia Math., 41 (1972) pp. 149-161.

[3] W. B. Johnson, Marlauschevioh bases and duality theory, Trans. Auner. Math. Soc., 149 (1970), pp. 1-7.

[4] A. Pietsch, Über die Erzeungung von (F')-Täumen duroh selbstalinungierte Operatoren, Math. Ann., 164 (1966), pp. 219-224.

[5] I. Singer, Bases in Banaoh spaces, Heidelberg 1970.

INSTYTUT MATEMATYOZNY POLSKIHJ AKADHMTI NAUK

\section{On operator-valued solutions of d'Alembert's functional equation, II}

by

J. KISYŃSKI (Warszawa)

Abstract. In the paper several negative examples are given, connected with the problem of representation of a cosine operator function $\mathscr{C}(t)$ in the form $\mathscr{C}(t)$ $=\frac{1}{2} \mathscr{G}(t)+\frac{1}{2} \mathscr{G}(-t)$, where $\mathscr{G}(t)$ is an one parameter group of operators.

Introduction and results. Let $X$ be a real or complex topological vector space and let $\mathscr{L}_{s}(X)$ be the space of all linear continuous operators of $X$ into itself with the topology of simple convergence. A continuous mapping $\mathscr{C}$ of $(-\infty, \infty)$ into $\mathscr{L}_{s}(X)$ is called the cosine operator function if it satisfies the d'Alembert functional equation

$$
\mathscr{C}(t+s)+\mathscr{C}(t-s)=2 \mathscr{C}(t) \mathscr{C}(s), \quad-\infty<s, t<\infty
$$

and if, moreover,

$$
\mathscr{C}(0)=1
$$

We shall say that $\mathscr{C}$ has an exponential representation if there is a oneparameter continuous group $\{\mathscr{G}(t):-\infty<t<\infty\} \subset \mathscr{L}_{s}(X)$ such that

$$
\mathscr{C}(t)=\frac{1}{2} \mathscr{G}(t)+\frac{1}{2} \mathscr{G}(-t), \quad-\infty<t<\infty .
$$

It was proved in [5] that if $X$ is a complex Banach space and an $\mathscr{L}(X)$-valued cosine function is bounded on $(-\infty, \infty)$ and continuous in the sence of the norm in $\mathscr{L}(X)$, then this cosine function has an exponential representation. Without the assumption of continuity in the sense of norm in $\mathscr{L}(X)$ a similar theorem is not true. Namely, as shown in [5], if $X$ is the space of all complex impair continuous functions on $(-\infty, \infty)$ having period $2 \pi$, or if $X$ is the space of all complex impair functions almost periodic in the sense of Bohr, and if

$$
(\mathscr{C}(t) x)(s)=\frac{1}{2} x(s+t)+\frac{1}{2} x(s-t), \quad x \in \mathcal{X},-\infty<s, t<\infty,
$$

then $\mathscr{C}$ has no exponential representation.

In the present paper some other examples of this type will be presented and the results may be summarized as follows. Consider following complex functional spaces on $(-\infty, \infty)$ : 